\title{
Switching Periodic Membranes via Pattern Transformation and Shape Memory Effect
}

\section{Citation}

Li, Jie, Jongmin Shim, Justin Deng, Johannes T. B. Overvelde, Xuelian Zhu, Katia Bertoldi, and Shu Yang. 2012. Switching periodic membranes via pattern transformation and shape memory effect. Soft Matter 8(40): 10322-10328.

\section{Published Version}

doi:10.1039/c2sm25816a

\section{Permanent link}

http://nrs.harvard.edu/urn-3:HUL.InstRepos:11130518

\section{Terms of Use}

This article was downloaded from Harvard University's DASH repository, and is made available under the terms and conditions applicable to Open Access Policy Articles, as set forth at http:// nrs.harvard.edu/urn-3:HUL.InstRepos:dash.current.terms-of-use\#OAP

\section{Share Your Story}

The Harvard community has made this article openly available.

Please share how this access benefits you. Submit a story.

Accessibility 


\title{
Switching Periodic Membranes via Pattern Transformation and Shape Memory Effect
}

\author{
Jie Li, ${ }^{a}$ Jongmin Shim, ${ }^{b}$ Justin Deng, ${ }^{c}$ Johannes T. B. Overvelde, ${ }^{b}$ Xuelian Zhu, ${ }^{a}$ Katia Bertoldi ${ }^{b}$ and \\ Shu Yang*a
}

\author{
Received (in $X X X, X X X) X$ th $X X X X X X X X X 20 X X$, Accepted Xth $X X X X X X X X X 20 X X$ \\ DOI: $10.1039 / b 000000 x$
}

10

\begin{abstract}
We exploited mechanical instability in shape memory polymer (SMP) membranes consisting of a hexagonal array of micron-sized circular holes and demonstrated dramatic color switching as a result of pattern transformation. When hot-pressed, the membrane underwent pattern transformation to an array of elliptical slits (with width of tens of nanometers) to featureless on surface with

15 increasing applied strain, switching the membrane with diffraction color to a transparent film. The deformed pattern and the resulting color change can be fixed at room temperature, both of which could be recovered upon reheating. Using continuum mechanical analyses, we modeled the pattern transformation and recovery processes, including the deformation, the cooling step, and the complete recovery of the microstructure, which corroborated well with experimental observations. We find that the elastic energy is roughly two-orders of magnitude larger than the surface energy in our system, leading to autonomous recovery of the structural color upon reheating. Furthermore, we demonstrated two potential applications of the color switching in the SMP periodic membranes by 1) temporarily erasing a pre-fabricated "Penn" logo in the film via hot-pressing, and 2) temporarily displaying "Penn" logo by hot-pressing the film against a stamp. In both scenarios, the original color

\section{Introduction}

Shape memory polymers (SMPs) are polymeric smart materials of interest for a variety of applications, including deployable space structures, artificial muscles, biomedical 30 devices, sensors, smart dry adhesives, and fasteners. ${ }^{1,2}$ They form a "permanent" shape by chemical or physical crosslinking (e.g. crystallization or chain entanglement). Above a thermal phase transition temperature, either a glass transition temperature $\left(T_{\mathrm{g}}\right)$ or a melting temperature $\left(T_{\mathrm{m}}\right)$,

35 SMPs can be deformed to different temporary shapes, which can be fixed by cooling the sample. Upon exposure to an external stimulus, such as heat, light, and solvent, the temporary shapes can return to their original (or the permanent) shape. There has been much effort to develop new

40 chemistry for improved shape fixity and shape recovery efficiency, responsiveness to new environmental triggers, achieving multi-shape memory effect, and applications to biomedical devices. ${ }^{1,3-9}$ Nevertheless, most of the study focuses on shape memory effect in bulk SMPs. A few groups

45 have created micropatterns in SMPs, such as microprotutions ${ }^{10}$ and microwrinkles ${ }^{8,11}$ by taking advantage of the large modulus change near the phase transition temperature. None of them, however, have reported the recovery to the original shape from the micropatterns. During 50 the shape recovery process, the entropic energy stored in the deformed state is released. It remains to be seen whether the deformed shape can be completely recovered as surface energy becomes increasingly dominant when the size shrinks to micro- and nanoscale.

55 Recently, we and several other groups have demonstrated pattern transformation in elastic membranes with periodic hole arrays by mechanical compression, ${ }^{12,}{ }^{13}$ solvent swelling, ${ }^{14,15}$ polymerization, ${ }^{16}$ and capillary force. ${ }^{17}$ For example, when swollen by an organic solvent, a 60 poly(dimethylsiloxane) (PDMS) membrane consisting of micron-sized circular holes in a square array buckles to a diamond plate pattern of elliptic slits with the neighboring units perpendicular to each other. ${ }^{14}$ As a result, the physical properties (e.g. photonic $^{18,19}$ and phononic ${ }^{15}$ band gap $^{18,19}$ 65 and mechanical behaviors ${ }^{20,21}$ ) could be significantly altered due to change of lattice symmetry, pore size, shape and volume filling fraction. One question rises whether it is possible to switch a colorful film to transparent one via pattern transformation. The latter state will allow for seeing 70 through or mingling with the surroundings. Therefore, the 


\title{
Cite this: DOI: $10.1039 / \mathrm{c0xx} 00000 \mathrm{x}$
}

\author{
www.rsc.org/xxxxxx
}

5 dramatic visual contrast between colored and transparent states is of interest for applications such as display, privacy window, and camouflage. In nature, invisibility is an important strategy for many sea creatures to hide from predators in water. For example, bobtail squids are invisible

10 in sand during the day with chromatophores in the skin concentrated into small, barely visible dots; when the muscle fibers stretch out the skin, thereby enlarging the chromatophores, the color becomes visible for signaling or escape from predators. ${ }^{22}$

15 Here we report switching a SMP membrane with diffraction color to a transparent film via harnessing the mechanical instability and shape memory effect. When hot-pressed, the SMP membrane consisting of a hexagonal array of circular holes $(1.2 \mu \mathrm{m}$ in diameter, $2.5 \mu \mathrm{m}$ in pitch,

20 and $5.0 \mu \mathrm{m}$ in depth) underwent pattern transformation to an array of elliptical slits to featureless on surface with increasing applied strain, leading to the dramatic change of the hole size and shape, and diffraction color, which could be fixed at room temperature, and later recovered to the original

25 pattern (and color) upon reheating. Using continuum mechanical analyses, we modeled, for the first time, an out-of-plane compression of SMP membrane. We observed the hot-press induced deformation and pattern transformation of the membrane at different strains, the structure fixation at

30 the cooling step, and the complete recovery of the microstructure, in agreement with experiments. We also find that the elastic energy stored in the membrane is roughly 2-orders of magnitude larger than the surface energy, leading to autonomous recovery of the structural color upon

35 reheating. Further, we demonstrated two possible applications of color and transparency change in our SMP periodic membranes, including 1) temporary erasing the pre-fabricated "Penn" logo in the film, and 2) a temporary display of "Penn" logo by hot-pressing the film against a stamp.

\section{Results and Discussion}

The ability to simultaneously change the lattice symmetry, pore size and shape, and volume filling fraction through pattern transformation offers an attractive approach to drastically alter the materials properties. Most deformation 45 methods reported so far involves the use of solvent, either through swelling or drying processes. In comparison, application of mechanical force will allow us to independently control the amount, direction (uniaxial or biaxial both in-plane and out-of-plane), and timing of strain

50 applied to the periodic structures. In the case of in-plane compression, however, additional care has to be taken to eliminate the out-of-plane buckling, e.g. by sandwiching the

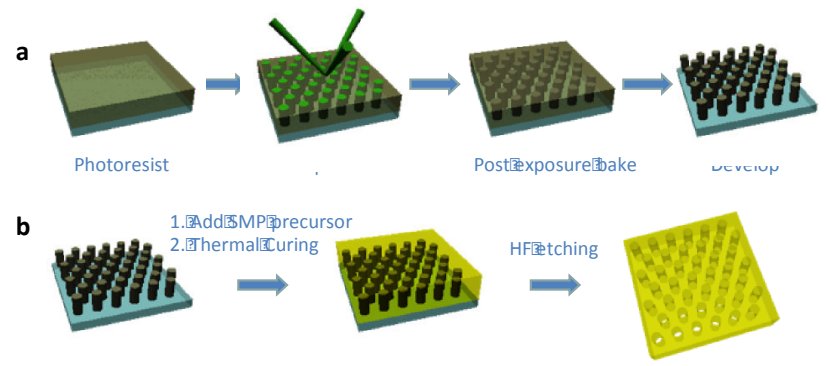

c

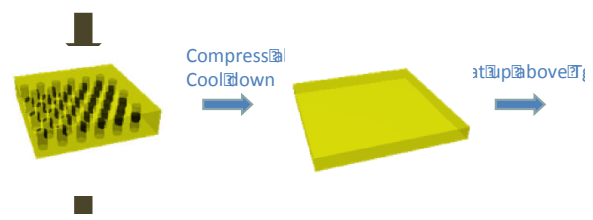

Fig.1 Schematic illustration of fabrication and 55 deformation/recovery of a 2D SMP membrane. (a) Fabrication of 2D pillar array by holographic lithography. (b) Fabrication of SMP membrane with periodic holes by replica molding, followed by etching in HF aqueous solution. (c) Hot-pressing of SMP membrane in the vertical direction above $T_{\mathrm{g}}$. The temporary shape could be

60 fixed by cooling down to room temperature under the load. The original shape could be recovered upon reheating above $T_{\mathrm{g}}$.

film between two rigid sheets. ${ }^{12}$ In most applications, a direct out-of-plane compression is easy to implement and desirable, and was thus performed in our experiments.

65 Color / Transparency Switching in SMP periodic membranes

The SMP periodic membrane $(1.2 \mu \mathrm{m}$ in diameter, $2.5 \mu \mathrm{m}$ in pitch, and $5 \mu \mathrm{m}$ in depth) was prepared by replica-molding from a 2D hexagonal pillar array, which was fabricated by 70 3-beam holographic lithography ${ }^{23,24}$ (see Fig. 1a-b and details in Experimental section). The negative-tone photoresist, epoxycyclohexyl POSS $\AA$ cage mixture (epoxy POSS) was chosen here to fabricate the pillar array since it could be readily removed by hydrofluoric acid (HF) solution at room

75 temperature $^{23}$ after templating the SMP membrane. When the latter was heated to $10-30{ }^{\circ} \mathrm{C}$ above its $T_{\mathrm{g}}\left(70{ }^{\circ} \mathrm{C}\right)$, it became softened and was compressed vertically by a hot-press to a temporary shape (Fig. 1c). The load was carefully controlled to deform the membrane at different strain levels, here

80 referring to engineering strain, $\varepsilon=$ change of film thickness/original thickness. The temporary shape was fixed when cooled down to room temperature while keeping the loading force constant. Upon reheating to $90{ }^{\circ} \mathrm{C}$, the hexagonal shape was recovered. During the pattern

85 deformation and recovery, we observed reversible switching of color and transparency.

Although the bulk SMP film is transparent, the SMP 


\title{
Cite this: DOI: $10.1039 / \mathrm{c0xx00000x}$
}

\author{
www.rsc.org/xxxxxx
}

Paper

5 membrane is colorful due to the diffraction grating effect

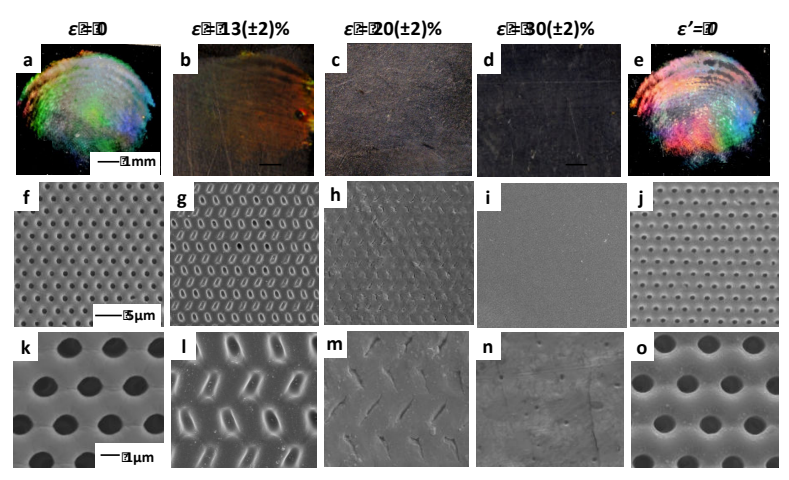

Fig. 2 Pattern transformation and recovery in a 2D SMP membrane. (a-e) Optical images of the (a) original, (b-c) partially deformed, $\varepsilon^{\sim}$ $13 \pm 2 \%$ (b) and $\varepsilon \sim 20 \pm 2 \%$ (c), (d) completely deformed, $\varepsilon \sim 30 \pm 2 \%$, 10 and (e) recovered SMP membranes. (f-j) Corresponding SEM images of the SMP membranes shown in (a-e). (k-o) Higher magnification SEM images of $(f-j)$.

(Fig. 2a, f, k). Because of the Gaussian distribution of the laser beam in holographic lithography and possible small 15 misalignment of optics, there was gradient laser intensity from center to the edge, resulting in pore size distribution and color variation across the sample size. This can be improved using a beam shaper or patterning the film by conventional photolithography through a photomask. When the applied

20 strain, $\varepsilon$, was $\sim 13 \pm 2 \%$, the circular holes of p6mm symmetry were deformed to elliptical slits (width of major axis, 1.25 $\mu \mathrm{m}$, minor axis, $500 \mathrm{~nm}$ ) with $p 2 g g$ symmetry (Fig $2 \mathrm{~g}, 1$ ), in agreement with the observation from the swelling-induced instability in SU-8 membranes with a hexagonal array of 25 pores. ${ }^{15}$ When the SMP membrane was compressed in the vertical direction, it expanded in-plane due to positive Poisson's ratio, hence generating an equivalent in-plane compressive stress to the circular holes. The initial diffraction color diminished significantly after compression although it 30 was not completely lost at this strain level (Fig. 2b). This could be attributed to the smaller pore size and porosity. The width of the minor axes of the ellipse further decreased, from hundreds of nanometers to a few nanometers, as the strain was increased. When $\varepsilon$ was increased to $\sim 20 \pm 2 \%$, the holes were 35 almost closed into lines (see Fig. 2c, h and $\mathrm{m}$ ) and the SMP membrane became quite transparent, much like the bulk film. At $\varepsilon \sim 30 \pm 2 \%$, the holes were closed-up and the surface became nearly featureless (Fig. 2d, i and n). No further change of transparency was observed. When any of the above

40 deformed SMP membranes were reheated to $90{ }^{\circ} \mathrm{C}$, the original periodic structure was restored nearly to completion
(97.6\% of the original hole size and $100 \%$ of the original pitch), as evident by the SEM images and the regeneration of strong diffraction color (Fig. 2e, j and o and Movie S1 $\dagger$ ).

45 Surprisingly, even the one with completely closed pores was restored, suggesting that the adhesive energy between the pore surfaces was much smaller than the elastic recovery energy. The different color displayed in Fig. 2a (the original film) and $2 \mathrm{e}$ (the recovered one) could be caused by a small 50 misalignment of incident light during photo shooting could lead to appearance of a different color. When $\varepsilon$ was greater than $50 \%$, the $2 \mathrm{D}$ grating with air holes and its color could no longer be completely recovered due to the permanent deformation of the polymer network.

The reversible switching between the colorful displays to transparency was repeated successfully for more than 10 cycles with $\varepsilon<50 \%$, and the recovery of diffraction color occurred within a few seconds (see Movie S1). According to SEM images, the hole diameter and pitch of the recovered 60 film decreased slightly to $94.4 \%$ and $98.4 \%$ of the original one after three cycles, respectively, and to $89.7 \%$ and $98.0 \%$ of the original one after ten cycles, respectively. The diffraction color displayed at any of the temporary state could be reprogrammed on demand by precise control of the applied 65 strain level and temperature/load of deformation. Hence, it is possible to build a color spectrum by carefully tuning the mechanical deformation. Further, we may achieve full-color display by combining the instability and design of the original microstructures with variable structural parameters.

70 During the pattern transformation and recovery process, the air holes were squeezed out and restored, respectively, which would result in a dramatic transparency change. As a proof-of-concept, we placed two SMP membranes on a paper printed with "Penn" logos: one was hot-pressed at $\varepsilon \sim 30 \pm 2 \%$

75 (the left one), and the other was the original, non-deformed one (the right one, see illustration in Fig. 3a). Due to diffraction from the surface of the original membrane with pores in hexagonal array, the "Penn" letters beneath it could not be clearly viewed, in sharp contrast to that beneath the

80 deformed membrane (see Fig. 3b). The transparence change was further investigated by UV-Vis spectroscopy at different thermal and mechanical treatments (Fig. 3c) using the bulk SMP film as a reference. As expected, the original sample (A) has the lowest transmittance (e.g. $28.1 \%$ at $\lambda=600 \mathrm{~nm}$ ). For 85 the hot-pressed samples, sample (B) that was deformed at $\varepsilon \sim$ $13 \pm 2 \%$ shows improved transmittance, $46.9 \%$ at $600 \mathrm{~nm}$, and the sample (C), which was deformed at $\varepsilon \sim 30 \pm 2 \%$ with closed voids, has the highest transmittance, $88.8 \%$ at $600 \mathrm{~nm}$, in comparison with the bulk SMP film The slightly lowered 90 transparency may be attributed to the surface roughness of the SMP membrane introduced by the Teflon sheets and dust 


\section{Cite this: DOI: $10.1039 / \mathrm{c0xx} 00000 x$}

\section{www.rsc.org/xxxxxx}

Paper

5 particles trapped on

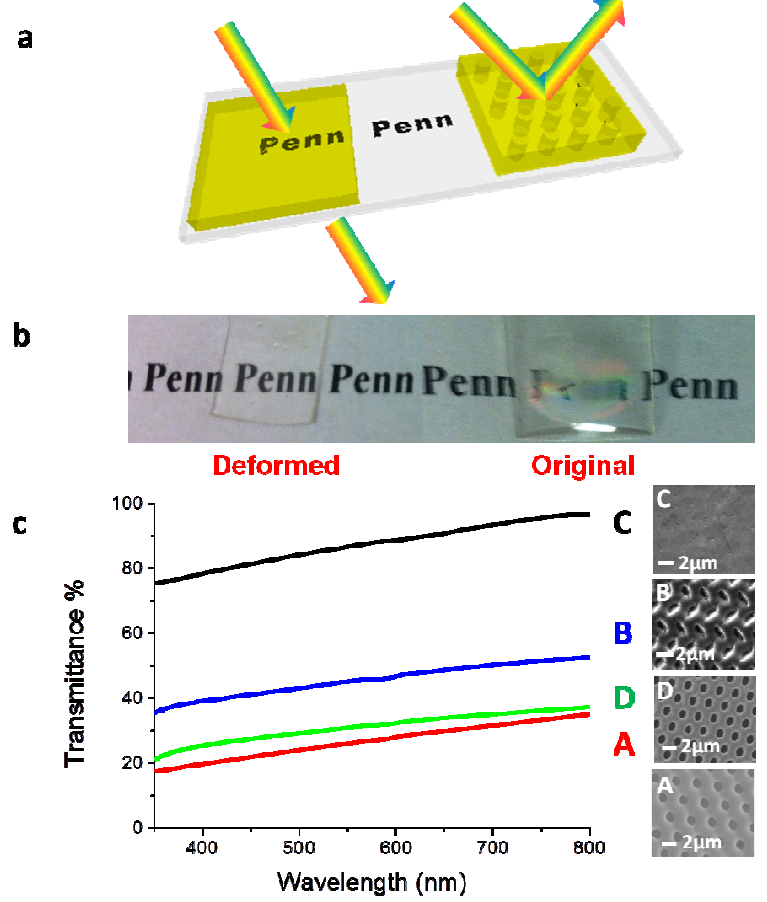

Fig. 3 Display of "Penn" logos underneath 2D SMP membranes. a. Schematic illustration of transparency comparison between the deformed and the original SMP membranes. b. Optical images of 10 the deformed and original SMP membranes on top of the "Penn" logo. The "Penn" underneath the original membrane is hardly legible but clearly visible under the deformed sample. c. UV-Vis spectra of different 2D SMP membranes (A-D) using the bulk SMP film as a reference. A. Original. B. Deformed at $\varepsilon \sim 13 \pm 2 \%$. C.

15 Deformed at $\varepsilon \sim 30 \pm 2 \%$. D. Recovered one.

the sample surface during hot-pressing and press release. Finally, the recovered sample (D) shows low transmittance $(32.5 \%$ at $600 \mathrm{~nm})$, close to that of the original membrane in the UV-visible region.

\section{Finite Element Analysis}

Since the deformation results presented here are the first demonstration of instabilities induced by loading in the direction perpendicular to the voids, we built a 3D mechanical

25 model to quantitatively investigate the buckling and post-buckling behaviors. The structure is modeled as an infinite array of infinitely long voids in the $\mathrm{x}_{1}-\mathrm{x}_{2}$ plane. 3D analyses are conducted and the constraining effect given by the substrate is accounted for by setting the lateral expansion

30 equal to zero. A periodic representative volume element

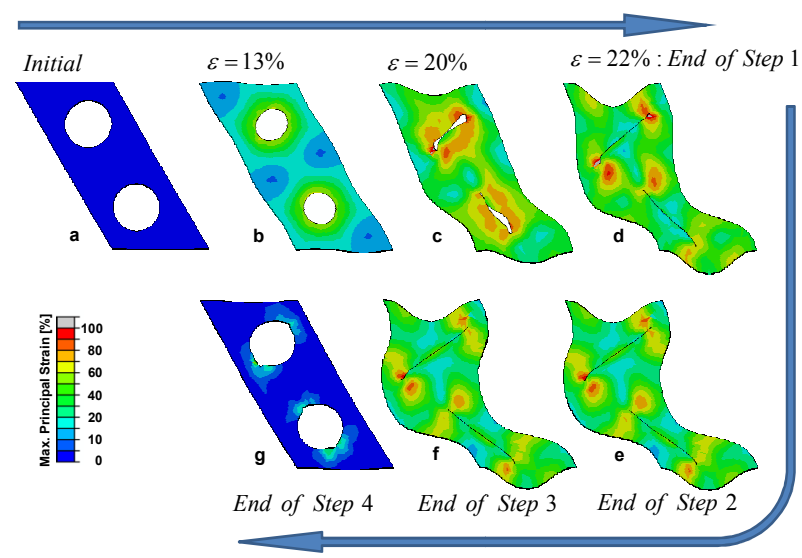

Fig. 4 Numerical results for the SMP thermo-mechanical cycle. Snapshots of the hexagonal lattice during Step 1, hot-pressing at different strain levels (a-d), and at the end of Steps 2, cooling down

40 (e), 3, un-loading (f) and 4, reheating (g). The color gives indications of the maximum principal strain distribution.

40 (RVE), as shown in Fig 4a, is considered and a series of constraint equations are applied to the boundaries of the model providing general periodic boundary conditions.

The stress-strain behavior of the SMP is captured using a two-mechanism constitutive model. ${ }^{15}$ The stress response is decomposed into two contributions: the resistance due to 50 stretching and orientation of the molecular network $\left(\sigma_{N}\right)$, mechanism $N$, and the resistance due to intermolecular interactions $\left(\sigma_{\mathrm{v}}\right)$, mechanism $V$. At the applied temperature $T$, the total stress acting on the material is given by

$$
\sigma=\sigma_{N}+V_{\mathrm{v}} \sigma_{\mathrm{v}}
$$

where

$$
v_{v}=1-\frac{1}{1+\exp \left[-\frac{T-T_{g}+A_{1}}{A_{2}}\right]} \text { with } A_{1} \text { and } A_{2} \text { as }
$$

material parameters defining the position and width of the zone where mechanism $V$ becomes significant.

The shape memory behavior is taken into account by having $\sigma_{\mathrm{v}}$ depend on $\left(T-T_{\mathrm{g}}\right)$. When $T>T_{\mathrm{g}}$ the material is characterized by a rubbery behavior; as $T$ decreases toward $T_{\mathrm{g}}$, the material becomes increasingly glassy and locked into the deformation.

65 The constitutive model is implemented into a user-defined subroutine (VUMAT) of the commercial finite element code ABAQUS, and numerical simulations of the whole thermo-mechanical loading history of the structures are performed in four steps (see Fig. 4 and Movie S2) using the 70 model parameters summarized in Table 1. 


\title{
Cite this: DOI: $10.1039 / \mathrm{c} 0 x x 00000 x$
}

\author{
www.rsc.org/xxxxxx
}

Paper

$5 \quad$ Step 1) Hot-pressing. $T$ increases above $T_{\mathrm{g}}$, so $\sigma_{\mathrm{v}}$ vanishes and the material exhibits rubber-like behavior. The stability Table 1. Material constants for SMP used in the mechanical models.

$$
\begin{array}{ccc}
\begin{array}{c}
\text { Rubbery Phase } \\
\text { (Mechanism N) }
\end{array} & \begin{array}{c}
\text { Glassy Phase } \\
(\text { Mechanism V) }
\end{array} & \begin{array}{c}
\text { Temperature } \\
\text { Related Constants }
\end{array} \\
& E=1500 \mathrm{MPa} \\
& v=0.45 & \\
\mu=1.72 \mathrm{MPa} & \dot{\gamma}_{0}=52 \times 10^{6} / \mathrm{sec} & T_{g}=343 \mathrm{~K} \\
N=4.33 & \Delta G=92 \times 10^{-21} \mathrm{~J} & A_{1}=20 \mathrm{~K} \\
K=16.7 \mathrm{MPa} & S_{0}=56 \mathrm{MPa} & A_{2}=5 \mathrm{~K} \\
& S_{S s}=28 \mathrm{MPa} & \\
& h=400 \mathrm{MPa} &
\end{array}
$$

$\mu$ is the elastic shear modulus, $N$ is the parameter relating to the limiting chain extensibility, $K$ is the bulk modulus, $E$ is the Young's 10 modulus, $v$ is Poisson's ratio, $\dot{\gamma}_{0}$ is pre-exponential shear strain rate factor, $\Delta G$ is activation energy, $s_{0}$ is the initial athermal deformation resistance, $s_{\mathrm{ss}}$ is the athermal deformation resistance value at the steady state, $h$ is the softening slope (the slope of the yield drop with respect to plastic strain).

15 of the structure is investigated by conducting a Bloch wave analysis. ${ }^{25}$ At an applied strain, $\varepsilon=11 \%$, a critical instability is detected, leading to the same pattern previously observed under constrained swelling, ${ }^{15}$ which is characterized by sheared voids where the shear direction alternates back and

20 forth from row to row (see Fig. 4a-c). Further compression leads to complete closure of the voids at $\varepsilon=22 \%$ (Fig. $4 d$ ), in agreement with experimental observation (Fig. 2h). In the simulations, further compression was avoided to prevent too much mesh distortion.

25 Step 2) Cooling down. $T$ decreases to $20{ }^{\circ} \mathrm{C}$, and $\sigma_{\mathrm{v}}$ increases, making the material much stiffer and preserving the pattern (Fig. 4e);

Step 3) Unloading. The press is removed, but the holes remain completely closed (Fig. 4f), and the elastic energy is stored in

30 the material;

Step 4) Reheating up. $T$ increases above $T_{\mathrm{g}}$ so that the structure again exhibits a rubbery behavior $\left(\sigma_{v}\right.$ vanishes again) and the initial shape and pattern are elastically recovered (see Fig. 4g).

As seen in Fig. 4, the numerical analysis nicely captured the deformation, pattern transformation and recovery of the SMP structures. Moreover, the analysis revealed a strong dependency of the onset of instability on the porosity of the structure. Thus the discrepancy between experiments and 40 simulations observed close to the onset of instability at $\varepsilon=$ $13 \%$ (Figs. $2 \mathrm{~g}$ and $4 \mathrm{~b}$ ) may be attributed to a small difference in porosity between the model and the tested

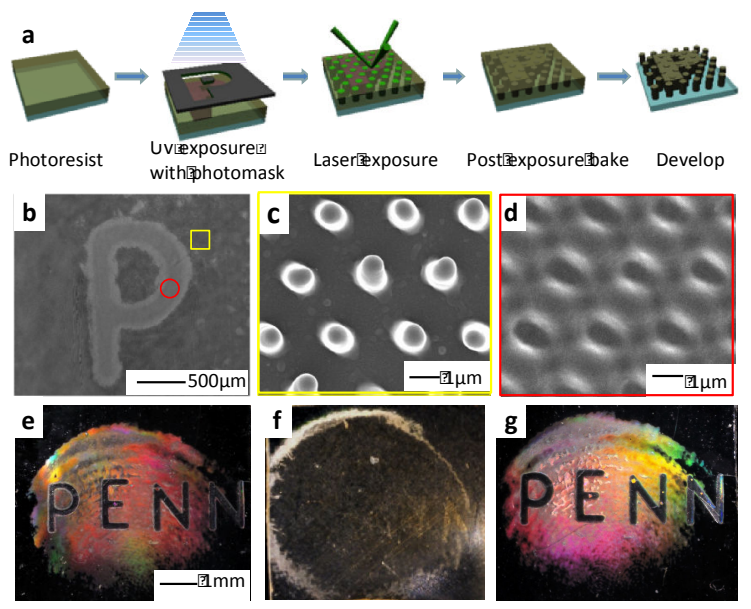

Fig. 5 Display of "PENN" embedded in a 2D SMP membrane. (a) 45 Schematic illustration of the fabrication of template. (b-d) SEM images of the photoresist template before replication to the SMP membrane. (b) A low magnification SEM image of the " $P$ " character in the template. (c) SEM image of the structure outside " $P$ " character, yellow square, showing tall pillars. (d) SEM image of the 50 structure inside the " $\mathrm{P}$ " character, red circle, showing nearly flat film with shallow voids. e-g. Optical images of the original (e), deformed ( $f$ ) and recovered (g) SMP films.

sample. Additionally, we find that for the considered structures with voids of $1 \mu \mathrm{m}$ in diameter the surface energy

$55\left(22.8 \mathrm{~mJ} / \mathrm{m}^{2}\right.$ measured by goniometer $)$ is roughly two orders magnitude smaller than the elastic recovery energy, making the recovery autonomous upon reheating. Since the strain energy is proportional to $L^{3}$ (with $L$ denoting the characteristic material dimension), while the surface energy is proportional $L^{2}$, a decrease of the voids diameter will increase the contribution of the surface energy. An approximate analysis suggests that the surface energy will play an important role for voids 10 times smaller than those considered in this study.

\section{Color displays with SMP periodic membranes}

65 To demonstrate the flexibility of color and transparency change in our SMP periodic membranes and their potential applications, we exploited two possible renderings of the SMP membranes. First, a "Penn" logo was pre-fabricated within the $2 \mathrm{D}$ membrane (Fig. 5a, b). The template for replica molding was fabricated by exposing the negative-tone photoresist, epoxy POSS, to UV light through a photomask with "Penn" logo, followed by 3-beam holographic 


\section{Cite this: DOI: $10.1039 / \mathrm{c0xx} 00000 \mathrm{x}$}

5 lithography to create hexagonal array of pillars in the surrounding area (Fig. 5c). Since the region with "Penn" was mostly crosslinked in the first step, the second exposure did not produce any pillar in this region but shallow voids (Fig. 5d). After replica-molding the template

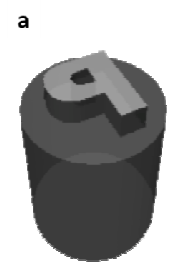

Fig.6 Indentation display on the SMP membrane. Schematic illustrations of (a) indentation of a stamp with a letter " $P$ " into a heated SMP membrane, (b) the display of letter " $\mathrm{P}$ " in the SMP membrane in the deformed region and (c) structural recovery upon 15 reheating. (d-e). Corresponding optical images of the indented "Penn" in the colored SMP membrane (d) and its erase after reheating the SMP membrane to $90{ }^{\circ} \mathrm{C}(\mathrm{e})$.

to SMP membrane, there was no or little color diffracted from this region in sharp contrast to the bright color from the

20 surrounding area (Fig. 5e, g). When the SMP membrane was hot-pressed above $T_{\mathrm{g}}$, "Penn" logo disappeared as the film became transparent (Fig. 5f). When reheated, the "Penn" logo reappeared together with its colorful background, confirming the success of shape recovery. Here, the logo was

25 pre-fabricated in the permanent shape, which could be temporarily erased upon deformation.

In a second approach, the "Penn" logo was introduced as a temporary shape by a rubber stamp indented into the SMP membrane during heating (Fig. 6a) at $90{ }^{\circ} \mathrm{C}$. The stamp was

30 released after the film was cooled down to room temperature. As seen in Fig. $6 b$ and $6 \mathrm{~d}$, the indented region was transparent, especially at the sharp corners of the letters, presumably receiving higher stress, while the background remained colorful. When reheated, the "Penn" logo was

35 erased (Fig. 6c, e). In this way, different letters or patterns could be "finger-printed" and reprogrammed into the same SMP membrane repeatedly, which could be extremely useful as a user-friendly touch screen display or fingerprinting by tailoring the SMP $T_{\mathrm{g}}$ near the body temperature. It should be

40 noted that all the displays presented here require no extra energy to maintain the displayed state.

\section{Conclusions}

We prepared 2D periodic membrane in SMPs, and studied the mechanical instability and shape memory effect. When hot-pressed, the membrane underwent pattern transformation from a $p 6 \mathrm{~mm}$ hexagonal lattice of circular holes $(1 \mu \mathrm{m}$ diameter) to a $p 2 g g$ pattern of elliptical slits (width varied from a few hundreds of $\mathrm{nm}$ to a few $\mathrm{nm}$ ), and eventually the

75 holes were completely closed. The original film is colorful because of the diffraction from the periodic micropattern and can be reversibly switched to a transparent state by mechanical deformation above the material's $\mathrm{T}_{\mathrm{g}}$. Upon reheating, the deformed patterns were able to recover, hence, 80 restoring the diffraction color. The combination of pattern transformation and shape memory effect in a $2 \mathrm{D}$ periodic membrane offers several distinctive characteristics. 1) It is the first demonstration of instabilities induced by loading in the direction perpendicular to the voids in microstructured SMPs, 85 which is more desirable in practical applications than approaches such as solvent swelling and in-plane compression. 2) The temporarily deformed structure and the resulting color can be fixed without the need for continuous input of external trigger; they can also be programmed 90 continuously by varying the mechanical strain level. 3) The continuum mechanical analyses have faithfully captured the buckling and post-buckling behaviors of the SMP membrane observed experimentally. Importantly, the model suggests that the surface energy plays a negligible role comparing with

95 elastic energy when the void dimension is comparable to the wavelength of light, leading to autonomous and fast shape recovery of the microstructure.

We emphasize that while the diffraction color change is 90 demonstrated in temperature responsive SMPs here, there are a broad range of stimuli responsive material systems in the literature, allowing for fine-tuning the transition temperature, switching speed, degree of responsiveness, number of temporary states, and the type of stimulus. For example, the

95 $T_{\mathrm{g}}$ of the epoxy SMP used in our system could be lowered (e.g. to $30{ }^{\circ} \mathrm{C}$ ) by increasing the concentration of more flexible crosslinker, decylamine. ${ }^{26}$ SMPs that can store up to three different shapes in temporary states have been reported. ${ }^{7,} 27 \mathrm{We}$ expect that the study of tuning periodic

100 structures via combined pattern transformation and shape memory effect will shed new light in harnessing the mechanical response of soft materials and advancing a wide range of technologies, including color displays, sensors, camouflage, and energy efficient building components (e.g. 5 smart windows and responsive façade).

\section{Experimental section}

Unless specifically noted, all chemicals were obtained from Sigma-Aldrich (St. Louis, MO, USA) and used as received. 


\section{Cite this: DOI: $10.1039 / \mathrm{c0xx} 00000 \mathrm{x}$}

\section{Fabrication of the hexagonal pillar array (Fig. 1a)}

The SMP periodic membrane was replica molded from a 2D hexagonal pillar array $(1.2 \mu \mathrm{m}$ in diameter, $2.5 \mu \mathrm{m}$ in pitch, and $5 \mu \mathrm{m}$ in height), which was fabricated by 3-beam holographic lithography (HL) $)^{23,24}$ from epoxycyclohexyl

10 POSS $®$ cage mixture (EP0408, Hybrid Plastics ${ }^{\circledR}$ ) (epoxy POSS) mixed with 0.9 wt \% photoinitiator, Irgacure 261 (Ciba Specialty Chemicals). In a typical HL experiment, the epoxy POSS photoresist was spin-coated on a glass substrate, prebaked at $50{ }^{\circ} \mathrm{C}$ for $40 \mathrm{~min}$, followed by $95{ }^{\circ} \mathrm{C}$ for $2 \mathrm{~min}$.

15 The film was then exposed to three interfering laser beams ( $\lambda=532 \mathrm{~nm}$, power of beam source $\sim 1.0 \mathrm{~W}$ ), followed by post-exposure bake (PEB) at $50{ }^{\circ} \mathrm{C}$ for $30 \mathrm{~s}$ (Fig. 1a). The pillar structures were obtained after development in propylene glycol methyl ether acetate (PGMEA), rinsing in

20 isopropanol (IPA), followed by drying in critical point dryer (SAMDRI ${ }^{\circledR}$-PVT-3D, tousimis) from ethanol to prevent pillar collapse. The sample area was defined by the laser beam size, typically $\sim 1 \mathrm{~cm}$ in diameter. By varying the dosage of laser exposure and the PEB time and temperature, we obtained

25 holes size ranging from hundreds of nanometers to a few microns.

\section{Replica molding SMP periodic membrane (Fig. 1b)}

The SMP precursor, a mixture with molar ratio 5:1:3 of melted diglycidyl ether of bisphenol A epoxy (EPON 826),

30 poly(propylene glycol)bis(2-aminopropyl)ether (Jaffamine D-230) and decylamine (DA), was infiltrate into the template via capillarity at $50{ }^{\circ} \mathrm{C}$ followed by thermal curing at $100{ }^{\circ} \mathrm{C}$ for $1.5 \mathrm{~h}$ and $150^{\circ} \mathrm{C}$ for $1 \mathrm{~h}$, respectively. The $T_{\mathrm{g}}$ can be tuned from $40{ }^{\circ} \mathrm{C}$ to $90{ }^{\circ} \mathrm{C}$ by varying the ratio of difunctional D230 and monofunctional DA. ${ }^{26}$ Here, the SMP was formulated to have a $T_{\mathrm{g}}$ of $70{ }^{\circ} \mathrm{C}$. After crosslinking EPON 826, the epoxy POSS template and the glass substrate were etched away by aqueous HF solution (48\% wt.), resulting in a free-standing SMP membrane of a hexagonal array of holes on a $\sim 500 \mu \mathrm{m}$ 40 thick bulk film.

\section{Hot pressing of SMP membranes}

The SMP membrane was compressed in the vertical direction using a manual bench top heated hydraulic press (CARVER 4122, Carver, Inc). The sample ( $>0.4 \mathrm{~mm}$ thick) was placed 4 inside of a Teflon sample holder ( $0.4 \mathrm{~mm}$ thick), which was then pressed between two Teflon sheets with heated platens. The platens were pre-heated to $100{ }^{\circ} \mathrm{C}$ for $10 \mathrm{~min}$ to reach equilibrium. Then a pressure of $1000 \mathrm{psi}$ was applied to the sample and kept for 15 min before cooling down to room

50 temperature, followed by release of the pressure to lock the temporary shape. The strain was calculated by comparing the final film thickness with the original one.

\section{Fabrication of SMP membrane with embedded "Penn"} letter

55 The membrane was fabricated by replica molding in the way similar to that from the hexagonal POSS pillar array. One added step was UV exposure ( $\lambda=365 \mathrm{~nm}, 400 \mathrm{~mJ} / \mathrm{cm}^{2}, 97435$ Oriel Flood Exposure Source, Newport) through a "Penn" logo photomask conducted after prebaking and before the

60 three-beam laser exposure. After PEB, the "Penn" region was highly crosslinked and appeared nearly flat or with shallow features depending on the dosage, while the surrounding areas formed pillar structures.

\section{Calculation/modelling}

65 Numerical simulations of stability of the structure were conducted using the nonlinear finite element code ABAQUS/Standard (version 6.8-2) while the thermo-mechanical loading history of the structures was investigated utilizing the nonlinear finite element code 70 ABAQUS/Explicit (version 6.8-2). Each mesh was constructed of 8-node, linear, 3D elements (ABAQUS element type C3D8R). In the hexagonal array the voids have a radius $R=1 \mu \mathrm{m}$ and a unit cell spanned by the lattice vectors $\mathrm{A} 1=\left[\begin{array}{lll}2 & 0 & 0\end{array}\right] \mu \mathrm{m}$ and $\mathrm{A} 2=\left[\begin{array}{lll}1 & 1.732 & 0\end{array}\right] \mu \mathrm{m}$ and $\mathrm{A} 3=\left[\begin{array}{lll}0 & 0 & 0.1\end{array}\right]$

$75 \mu \mathrm{m}$ is used. RVE consisting of $1 \times 2 \times 1$ unit cells is considered in the simulations of the thermo-mechanical loading cycle and an imperfection in the form of the most critical eigenmode is introduced into the mesh to capture the instability upon hot-pressing, the subsequent freezing-in of the transformed 80 pattern and then the shape recovery behavior. The stress-strain behavior of the SMP is captured using the material parameters reported in Table 1.

\section{Acknowledgements}

The research is in part supported by the National Science 85 Foundation (NSF), grant \# CMMI-0900468 and EFRI-1038215, and University Research Foundation (URF) at University of Pennsylvania. J. D. would like to thank the Penn Materials Research Science and Engineering Center (DMR-0520020) REU program for support of his summer 90 research at University of Pennsylvania. K.B. and J.S. acknowledge the support of the Harvard Materials Research Science and Engineering Center under NSF award number DMR-0820484. Penn Regional Nanotech facility (PRFN) is acknowledged for access SEM. We are grateful to Prof. Karen 95 Winey for the use of the CARVER hot press, Dr. Tao Xie (General Motors Global Research \& Development) for 


\section{Cite this: DOI: $10.1039 / \mathrm{c0xx00000x}$}

5 helpful discussion of the use of the epoxy SMPs, and Mr. Felice Macera for helping with the photograph.

\section{Notes and reference}

${ }^{a}$ Department of Materials Science and Engineering, University of Pennsylvania, 3231 Walnut Street, Philadelphia, PA 19104, USA.

10 Fax: (+01)215-573-2128; Tel: (+01)215-898-9645; E-mail: shuyang@seas.upenn.edu

${ }^{b}$ School of Engineering and Applied Science, Harvard University, Cambridge, MA 02138,USA.E-mail:bertoldi@seas.harvard.edu

${ }^{c}$ Department of Materials Science and Engineering, Cornell

15 University, Bard Hall, Ithaca, NY 14853, USA.

$\dagger$ Electronic Supplementary Information (ESI) available: Movie showing the shape and color recovery of compressed, transparent SMP membrane after immersing in $90{ }^{\circ} \mathrm{C}$ water; movie showing FEM simulation of the pattern transformation of 2D SMP membrane

20 through compression and its recovery. See DOI: 10.1039/b000000x/

1. A. Lendlein and R. Langer, Science, 2002, 296, 1673.

2. C. Liu, H. Qin and P. T. Mather, J. Mater. Chem., 2007, 17, 1543.

3. A. Lendlein, H. Y. Jiang, O. Junger and R. Langer, Nature, 2005, 434, 879.

4. J. M. Ortega, W. Small, T. S. Wilson, W. J. Benett, J. M. Loge and D. J. Maitland, IEEE Trans. Biomed. Eng., 2007, 54, 1722.

5. C. M. Yakacki, R. Shandas, D. Safranski, A. M. Ortega, K. Sassaman and K. Gall, Adv. Funct. Mater., 2008, 18, 2428.

6. T. Chung, A. Rorno-Uribe and P. T. Mather, Macromolecules, 2008, 41, 184.

7. T. Xie, Nature, 2010, 464, 267.

8. T. Xie, X. C. Xiao, J. J. Li and R. M. Wang, Adv. Mater., 2010, 22, 4390.

35 9. L. Sun and W. M. Huang, Soft Matter, 2010, 6, 4403.

10. N. Liu, Q. Xie, W. M. Huang, S. J. Phee and N. Q. Guo, J. Micromech. Microeng., 2008, 18.

11. Y. Zhao, W. M. Huang and Y. Q. Fu, J. Micromech. Microeng., 2011, 21.

12. T. Mullin, S. Deschanel, K. Bertoldi and M. C. Boyce, Phys. Rev. Lett., 2007, 99, 084301.
13. S. Willshaw and T. Mullin, Soft Matter, 2012, 8, 1747.

14. Y. Zhang, E. A. Matsumoto, A. Peter, P. C. Lin, R. D. Kamien and S. Yang, Nano Lett., 2008, 8, 1192.

45 15. J. H. Jang, C. Y. Koh, K. Bertoldi, M. C. Boyce and E. L. Thomas, Nano Lett., 2009, 9, 2113.

16. S. Singamaneni, K. Bertoldi, S. Chang, J. H. Jang, E. L. Thomas, M. C. Boyce and V. V. Tsukruk, ACS Appl. Mater. Interfaces, 2009, 1, 42.

50 17. X. Zhu, G. Wu, R. Dong, C.-M. Chen and S. Yang, Soft Matter, $2012,8,8088$.

18. X. L. Zhu, Y. Zhang, D. Chandra, S. C. Cheng, J. M. Kikkawa and S. Yang, Appl. Phys. Lett., 2008, 93, 161911.

19. D. Krishnan and H. T. Johnson, J. Mech. Phys. Solids, 2009, 57, 1500.

20. S. Singamaneni, K. Bertoldi, S. Chang, J. H. Jang, S. L. Young, E. L. Thomas, M. C. Boyce and V. V. Tsukruk, Adv. Funct. Mater., 2009, 19, 1426.

21. K. Bertoldi, P. M. Reis, S. Willshaw and T. Mullin, Adv. Mater., 2010, 22, 361.

22. M. Izumi, A. M. Sweeney, D. DeMartini, J. C. Weaver, M. L. Powers, A. Tao, T. V. Silvas, R. M. Kramer, W. J. Crookes-Goodson, L. M. Mathger, R. R. Naik, R. T. Hanlon and D. E. Morse, J. R. Soc. Interface, 2010, 7, 549.

65 23. Y. Xu, X. Zhu and S. Yang, ACS nano, 2009, 3, 3251.

24. J. H. Moon, A. J. Kim, J. C. Crocker and S. Yang, Adv. Mater., 2007, 19, 2508.

25. K. Bertoldi, M. C. Boyce, S. Deschanel, S. M. Prange and T. Mullin, J. Mech. Phys. Solids, 2008, 56, 2642.

$70 \quad$ 26. T. Xie and I. A. Rousseau, Polymer, 2009, 50, 1852.

27. I. Bellin, S. Kelch, R. Langer and A. Lendlein, Proc. Natl. Acad. Sci. U. S. A., 2006, 103, 18043.

\section{TOC figure}

Two-dimensional periodic membranes fabricated from shape memory polymers undergo pattern transformation when hot-pressed above the glass transition temperature. The change of lattice symmetry, size and filling fraction leads to disappearance of the 
Cite this: DOI: $10.1039 / \mathrm{c} 0 x \times 00000 x$

www.rsc.org/xxxxxx

Paper

reflective structural color, which can be recovered upon reheating.

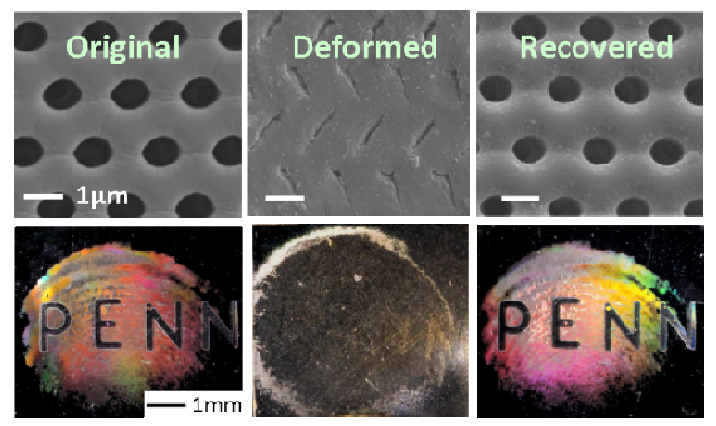

